\title{
Dependence of the viscoelastic response of the urinary bladder wall on strain rate
}

\section{R. van Mastrigt}

Department of Urology, Erasmus University Rotterdam, 3000 DR Rotterdam, The Netherlands

\section{J. C. Nagtegaal}

Department of Mechanical Engineering, Technical University Eindhoven, $5600 \mathrm{MB}$ Eindhoven, The Netheriands

\begin{abstract}
In order to relate the results of measurements on strips of urinary bladder wall to clinical measurements on the intact urinary bladder, an expression is derived for the dependence of the viscoelastic response of bladder-wallmaterial on strain rate. This expression is based on a non-linear viscoelastic model, with elastic moduli depending exponentially on strain, in accordance with the results of previous measurements. The results of measurements performed on strips of bladder-wall material at different strain rates show good agreement with the values predicted by the above mentioned expression.
\end{abstract}

Keywords -Bladder, Model, Rate of strain, Strain rate, Urinary bladder, Viscoelastic model

\section{Introduction}

THE PASSIVE properties of the urinary bladder in the collection phase can adequately be described in terms of a non-linear viscoelastic model (VAN MASTRIGT et al., 1978). The parameters of this model were determined mainly by applying stepwise straining to strips of bladder-wall material (VAN MASTRIGT, $1977 b$ ). It was concluded from consideration of this model that a good method of testing the passive properties of the urinary bladder for clinical purposes might be stepwise cystometry, involving very fast filling of the urinary bladder and observation of the resulting pressure decrease curve (COOLSAET et al., 1973). The rate at which the bladder wall is strained during stepwise cystometry is of course much lower than the rate at which strips of bladder wall can be strained. The results of stepwise cystometry must therefore be corrected for the lower strain rates involved. Since a simple correction formula based on a linear viscoelastic model (VAN MASTRIGT, 1977b) did not adequately describe viscoelastic responses at lower strain rates (VAN MASTRIGT et al., 1978) we decided to carry out a separate investigation of the strain-rate dependence of the viscoelastic response of the urinary bladder wall.

\section{Theory}

The viscoelastic model used is formed by combining a number of 'Maxwell' elements in parallel (see Fig. 1). A Maxwell element consists of a spring and a dashpot, both of which are assumed to be non-linear in our case, in series (see Fig. 2). The total strain $\varepsilon$ in the Maxwell

Received 3rd June 1980

$0140-0118 / 81 / 030291+06 \$ 01 \cdot 50 / 0$

(C) IFMBE: 1981 element is the sum of the strain in the spring $\varepsilon_{s}$ and that in the dashpot $\varepsilon_{d}$ :

$$
\varepsilon=\varepsilon_{\mathrm{s}}+\varepsilon_{d}
$$

Each strain component is defined as the change in length divided by the reference length $l_{0}$ of the overall Maxwell element. Now we assume that the constitutive equation of the spring has the form:

$$
\frac{\mathrm{d} \sigma}{\mathrm{d} t}=E_{\mathrm{t}}(\varepsilon) \frac{\mathrm{d} \varepsilon_{s}}{\mathrm{~d} t}
$$

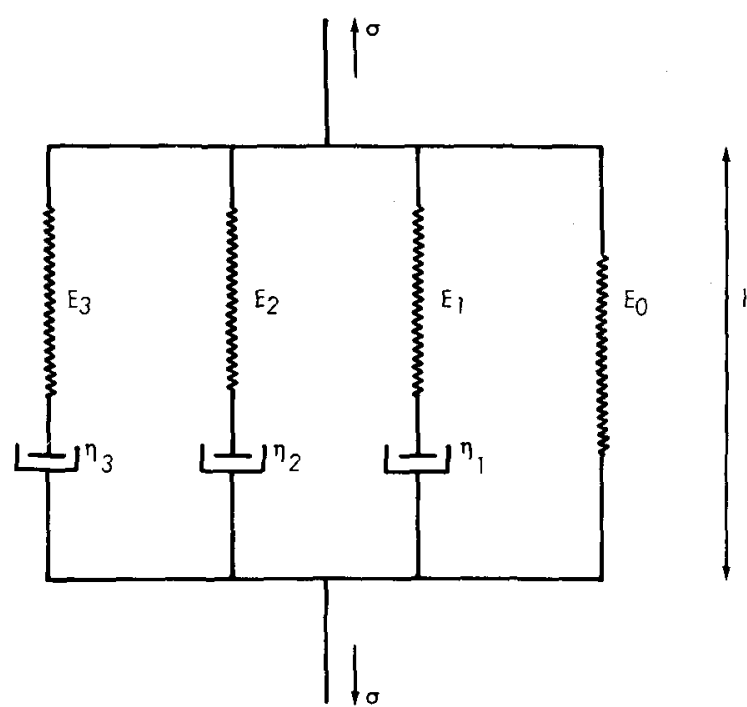

Fig. 1 Complete viscoelastic model consisting of three Maxwell elements and one purely elastic element in parallel 


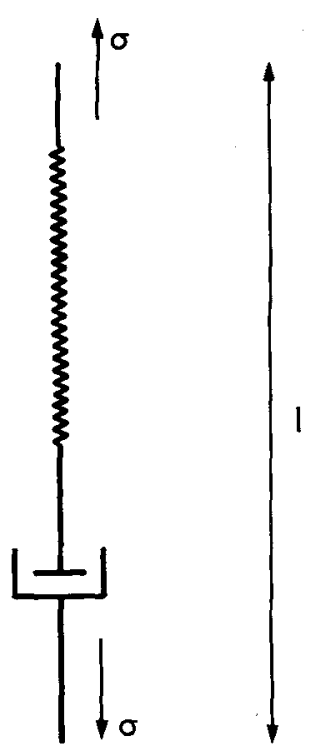

Fig. 2 Maxwell viscoelastic element, consisting of one elastic element and one viscous element in series

and that of the dashpot is:

$$
\sigma=\eta(\varepsilon) \frac{\mathrm{d} \varepsilon_{d}}{\mathrm{~d} t}
$$

In these equations $E_{t}$ is the 'tangent modulus' of the spring, see Fig. 3, $\sigma$ is the stress, expressed as force per unit area, and $\eta$ is the viscosity of the dashpot. It is assumed that both $E_{t}$ and $\eta$ depend on the total strain $\varepsilon$. Differentiation of eqn. 1 with respect to time and elimination of $\frac{\mathrm{d} \varepsilon_{\mathrm{s}}}{\mathrm{d} t}$ and $\frac{\mathrm{d} \varepsilon_{d}}{\mathrm{~d} t}$ yields the constitutive equation of the Maxwell element:

$$
\frac{\mathrm{d} \sigma}{\mathrm{d} t}=E_{t}(\varepsilon) \frac{\mathrm{d} \varepsilon}{\mathrm{d} t}-\frac{E_{t}(\varepsilon)}{\eta(\varepsilon)} \sigma
$$

Although both $E_{t}$ and $\eta$ depend on strain, it has been shown (VAN MASTRIGT et al., 1978) that the relaxation time of the element does not depend on strain. For the model, this means that the relaxation parameter:

$$
\gamma=\frac{E_{t}(\varepsilon)}{\eta(\varepsilon)}
$$

is a constant, which yields the final constitutive equation:

$$
\frac{\mathrm{d} \sigma}{\mathrm{d} t}=E_{t}(\varepsilon) \frac{\mathrm{d} \varepsilon}{\mathrm{d} t}-\gamma \sigma
$$

The solution of this differential equation yields:

$$
\sigma(t)=\mathrm{e}^{-; t} \int_{0}^{t} E_{t}(\varepsilon) \frac{\mathrm{d} \varepsilon(\tau)}{\mathrm{d} \tau} \mathrm{e}^{; \tau} \mathrm{d} \tau
$$

By straining the model stepwise as follows (see Fig. 4a):

$$
\varepsilon(\tau)=\varepsilon_{0} u(\tau)
$$

where $u(\tau)$ is the unity step function, we obtain as a response (see Fig. 4b):

$$
\sigma(t)=\mathrm{e}^{-r t} \int_{0}^{c 0} E_{t}(\varepsilon) \mathrm{d} \varepsilon=\sigma_{0}\left(\varepsilon_{0}\right) \mathrm{e}^{-\not t}
$$

where $\sigma_{0}\left(\varepsilon_{0}\right)$ is by definition the amplitude of the response to a stepwise strain of amplitude $\varepsilon_{0}$.

The result of straining the element more slowly up to the same amplitude is obtained by inserting:

$$
\begin{aligned}
& \varepsilon(\tau)=\varepsilon_{0} \cdot \frac{\tau}{t_{1}} \text { for } \tau>0 \text { (see Fig. } 4 c \text { ). } . \\
& \varepsilon(\tau)=0 \text { for } \tau \leqslant 0
\end{aligned}
$$

where $t_{1}$ is the time necessary to reach the desired strain level $\varepsilon_{0}$. Inserting the derivative of eqn. 10 into eqn. 7 and performing a partial integration, we obtain:

$$
\sigma\left(t_{1}\right)=\sigma_{0}\left(\varepsilon_{0}\right)-\mathrm{e}^{-\gamma t_{1}} \int_{0}^{\varepsilon_{0}} \sigma_{0}(\varepsilon) \frac{\gamma t_{1}}{\varepsilon_{0}} \mathrm{e}^{\gamma t_{1} \varepsilon / \varepsilon_{0}} \mathrm{~d} \varepsilon
$$

For $t>t_{1}$, where the linear increase in strain passes into a constant strain (see Fig. 4c), it can be seen directly from eqn. 6 by inserting $\frac{\mathrm{d} \varepsilon}{\mathrm{d} t}=0$ that viscoelastic relaxation as in eqn. 9 again follows, with the amplitude $\sigma_{0}\left(\varepsilon_{0}\right)$ replaced by eqn. 11 . We will therefore call $\frac{\sigma\left(t_{1}\right)}{\sigma_{0}\left(\varepsilon_{0}\right)}$ the 'factor' of the relaxation following a 'physical step' straining.

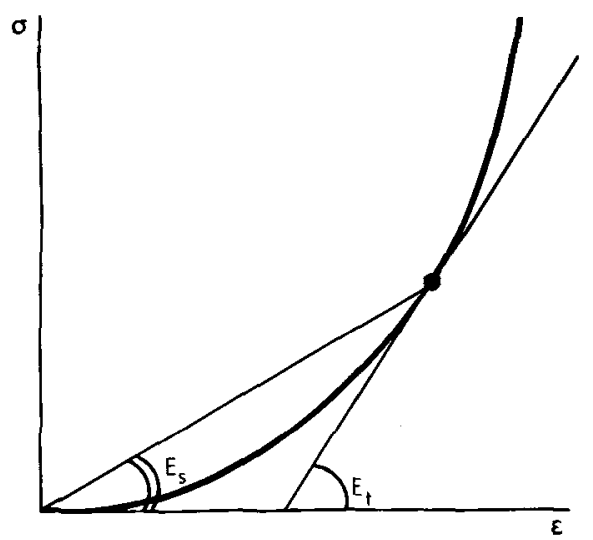

Fig. 3 Stress-strain curve for a non-linear spring, showing the principle of determination of the 'secant' and 'tangent' moduli 
In the previously published viscoelastic model (VAN MASTRIGT et al., 1978), the elastic modulus was defined in a different way, as the 'secant modulus' $E_{s}(\varepsilon)$, see Fig. 3.

The constitutive equation for a non-linear spring using the secant modulus reads:

$$
\sigma=E_{\mathrm{s}}(\varepsilon) \cdot \varepsilon_{\mathrm{s}}
$$

Since stepwise straining of the Maxwell element initially only strains the spring, we can write:

$$
\sigma_{0}(\varepsilon)=E_{s}(\varepsilon) \cdot \varepsilon
$$

Now if the model is linear, we may write:

$$
E_{\mathrm{s}}(\varepsilon)=E_{\mathrm{t}}(\varepsilon)=\mathrm{constan} t=E_{0} . \quad . \quad .
$$

Substituting eqns. 14 and 13 in eqn. 11 and partially integrating, we obtain:

$$
\sigma\left(t_{1}\right)=\sigma_{0}\left(\varepsilon_{0}\right)\left[\frac{1}{\gamma t_{1}}\left(1-\mathrm{e}^{-\gamma t_{1}}\right)\right] . \quad .
$$

This is the approximate formula obtained previously
(VAN MASTRIGT, 1977b) which is not applicable as will be shown.

If we replace eqn. 14 by:

$$
E_{s}(\varepsilon)=E_{0} \mathrm{e}^{\beta \varepsilon}
$$

(which was found to be the case in urinary bladderwall material (VAN MASTRIGT et al., 1978)), substitute this together with eqn. 13 in eqn. 11 and partially integrate, we find:

$$
\sigma\left(t_{1}\right)=\sigma_{0}\left(\varepsilon_{0}\right)\left[1-\psi+\frac{\psi^{2}}{\gamma t_{1}}\left(1-\mathrm{e}^{-(\xi t / \psi)}\right] .\right.
$$

where: $\psi=\frac{\xi}{\xi+1}$ and $\xi=\frac{\gamma t_{1}}{\beta \varepsilon_{0}}$ (see Fig. $4 d$ )

In fact, the urinary bladder-wall model used contains three Maxwell elements (Fig. 1) in parallel and the elastic modulus, eqn. 16 , is biexponential (VAN MASTRIGT et al., 1978).

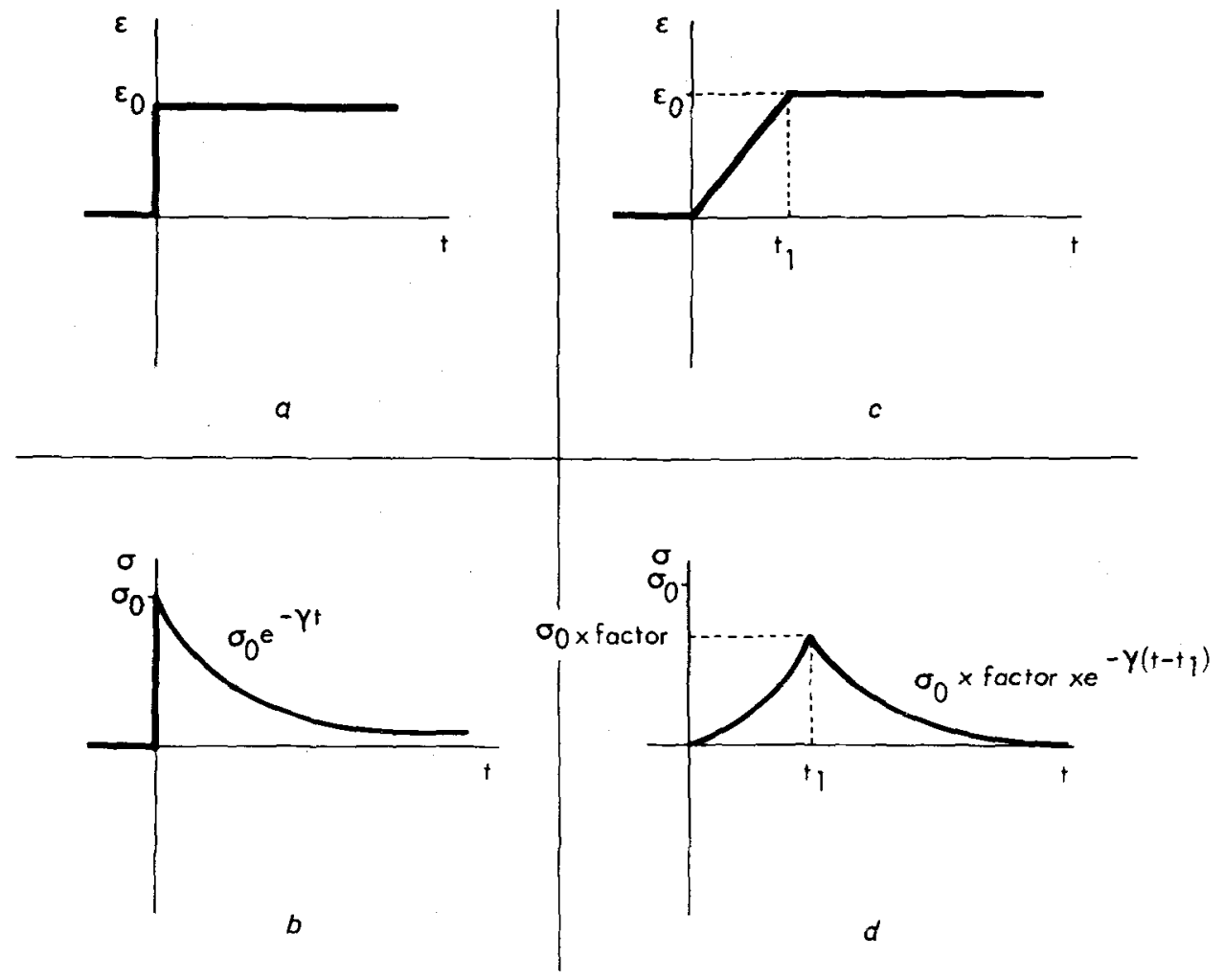

Fig. 4 (a) Stepwise strain

(b) Stress response of Maxwell element to stepwise
strain (c) Slower strain up to same amplitude as (a)

(d) Stress response of Maxwell element to slower strain 
The consequence of having three elements in parallel is that we have to add three forms like eqn. 17 with different values of $\gamma$ and different relaxation terms $\mathrm{e}^{-\%}$. By replacing eqn. 16 by a biexponential elastic modulus and recalculating eqn. 17 , we can easily show that this leads to a weighted addition of two forms of eqn. 17 with a different value of $\beta$ for each element.

It follows from the measured results given in Section 4 that only one of these forms is relevant.

Finally, the viscoelastic model mentioned above also contains a purely elastic element in parallel. This element is of course not affected by strain rates and adds a constant, non-decaying term to eqn. 17. The complete model is shown in Fig. 1.

\section{Methods}

Experiments were performed on pig urinary bladder-wall strips measuring about $10 \times 35 \mathrm{~mm}$, obtained from the local slaughterhouse.

The strips were mounted between two clamps with penetrating pins, the upper clamp being connected to a force transducer, while the lower clamp was slowly moved by paying out a steel wire connected to an infusion pump. Tension on the steel wire was maintained using a pneumatic cylinder. Except for this modification the apparatus was as described before (COOLSAET, 1976). The strips were immersed in a modified Krebs solution (COOLSAET, 1975), aerated with $95 \% \mathrm{O}_{2}$ and $5 \% \mathrm{CO}_{2}$ and kept at $37^{\circ} \mathrm{C}$. The strips were all strained up to a relative strain 0.5 (which means a $50 \%$ increase in length of the strip) at rates varying from 0.6 to $0.006 \mathrm{~mm} \mathrm{~s}^{-1}$, which means that the time necessary to reach full strain $t_{1}$ varied from 30 to $3000 \mathrm{~s}$. Measurements were also made at a practically infinite strain rate $\left(>1000 \mathrm{~mm} \mathrm{~s}^{-1}\right)$ using the pneumatic cylinder only. The resulting forces were read directly into a minicomputer for $1000 \mathrm{~s}$ after the attainment of the final level of 0.5 (i.e. during the relaxation phase).

These relaxation curves were fitted with a model of three exponential terms and a constant as follows:

$$
\sigma(t)=A_{0}+A_{1} \mathrm{e}^{-i t t}+A_{2} \mathrm{e}^{-i 2 t}+A_{3} \mathrm{e}^{-\% 3 t} .
$$

according to the viscoelastic model (Fig. 1) and eqns.
17 and 9. The fitting procedure has been described before (VAN MASTRIGT, 1977a). It gives seven parameters, viz three relaxation constants $\gamma_{1}, \gamma_{2}, \gamma_{3}$ three coefficients $A_{1}, A_{2}, A_{3}$ representing the intial heights of the exponential decay, and a constant $A_{0}$ corresponding to the simple spring in Fig. 1.

The coefficients $A_{i}$ were given the dimension of stress by dividing the measured forces by crosssectional area, calculated as the quotient of tissue volume and length of the strip, and thus equalled $\sigma\left(t_{1}\right)$ in eqn. 17 for every Maxwell element.

\section{Results}

Measurements were carried out on four strips. Eight measurements were made on every strip, two at an 'infinite' strain rate and six at lower speeds. The measurements were carried out in an alternating order, i.e. the first measurement was taken at a very high speed, the second at a low speed, etc. Table 1 gives average values and relative standard deviations

$$
\text { (i.e. } \left.\frac{\text { standard deviation }}{\text { average }} \times 100 \%\right)
$$

of the fitted relaxation constants as functions of strain rate. Some measurements at the lowest strain rate could not be successfully fitted with a threeexponential model, and at still lower strain rates $\left(0.006 \mathrm{~mm} \mathrm{~s}^{-1}\right)$ no successful measurements could be made at all.

It can be seen that the relaxation constants depend markedly on the strain rate. This effect, however, is not taken into account in the present model.

The non-decaying term $A_{0}$, corresponding to the spring without dashpot in Fig. 1, was found to be independent of strain rate as expected, i.e. it showed no appreciable variation in the measurements on one strip (relative standard deviation for the four strips $22 \%, 9 \%, 18 \% 15 \%) . A_{0}$ was therefore used to normalise the other coefficients $A_{1}, A_{2}, A_{3}$ in order to be able to compare the results for the different strips. Table 2 gives average values and relative standard deviations for the normalised coefficients $A_{1} / A_{0}, A_{2} / A_{0}, A_{3} / A_{0}$ as functions of strain rate. Table 1 . Average values and relative standard deviations $\left(\right.$ i.e. $\left.\frac{\text { standard deviation }}{\text { average }} \times 100 \%\right)$ of relaxation constants as functions of
strain rate

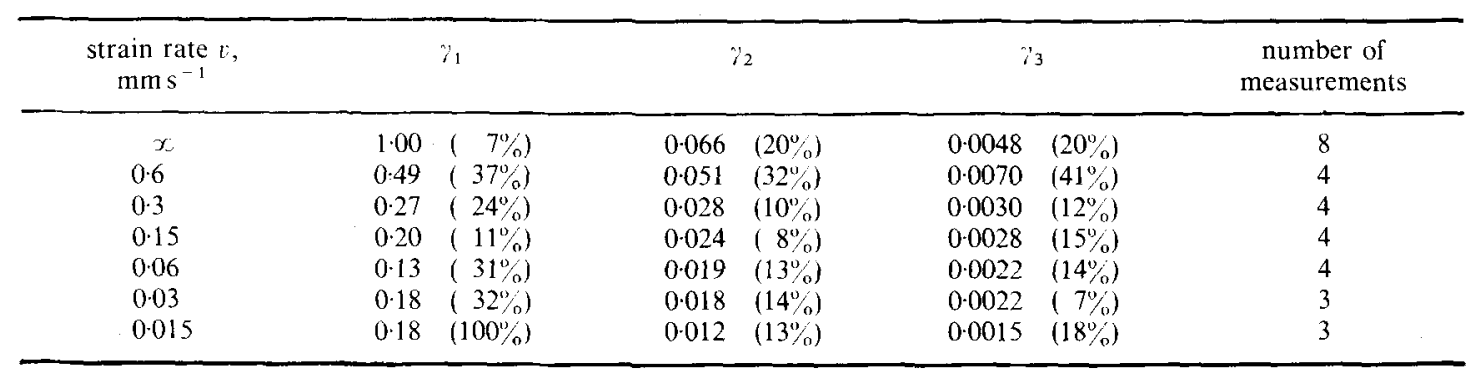


Since it has previously been found (VAN MASTRIGT et al., 1978) that all elastic moduli show the same strain
The broken line in Fig. 5 represents the approximate expression (eqn. 15) based on a linear model. It will be Table 2. Average values and relative standard deviations $\left(\right.$ i.e. $\left.\frac{\text { standard deviation }}{\text { average }} \times 100 \%\right)$ of the coefficients normalised with
respect to the constant $A_{0}$ as functions of strain rate

\begin{tabular}{|c|c|c|c|c|c|c|c|}
\hline \multirow{2}{*}{$\frac{\begin{array}{c}\text { strain rate } v \\
\mathrm{~mm} \mathrm{~s}^{-1}\end{array}}{\infty}$} & \multicolumn{2}{|c|}{$A_{\mathbf{1}} / A_{0}$} & \multicolumn{2}{|c|}{$A_{2} / A_{0}$} & \multicolumn{2}{|c|}{$A_{3} / A_{0}$} & \multirow{2}{*}{$\frac{\begin{array}{c}\text { number of } \\
\text { measurements }\end{array}}{8}$} \\
\hline & $2 \cdot 29$ & $(35 \%)$ & 0.79 & $(26 \%)$ & $0 \cdot 50$ & $(17 \%)$ & \\
\hline 0.6 & $0 \cdot 78$ & $(6 \%)$ & 0.62 & $(14 \%)$ & 0.48 & $(5 \%)$ & 4 \\
\hline $0 \cdot 3$ & 0.48 & $(13 \%)$ & 0.47 & $(14 \%)$ & 0.46 & $(20 \%)$ & 4 \\
\hline $0 \cdot 15$ & $0 \cdot 35$ & $(14 \%)$ & 0.42 & $(5 \%)$ & $0 \cdot 42$ & $(10 \%)$ & 4 \\
\hline 0.06 & 0.23 & $(22 \%)$ & $0 \cdot 36$ & $(21 \%)$ & $0 \cdot 46$ & $(25 \%)$ & 4 \\
\hline 0.03 & $0 \cdot 12$ & $(10 \%)$ & $0 \cdot 29$ & $(21 \%)$ & 0.42 & $(22 \%)$ & 3 \\
\hline $0 \cdot 016$ & $0 \cdot 10$ & $(43 \%)$ & $0 \cdot 28$ & $(19 \%)$ & $0 \cdot 48$ & $(4 \%)$ & 3 \\
\hline
\end{tabular}

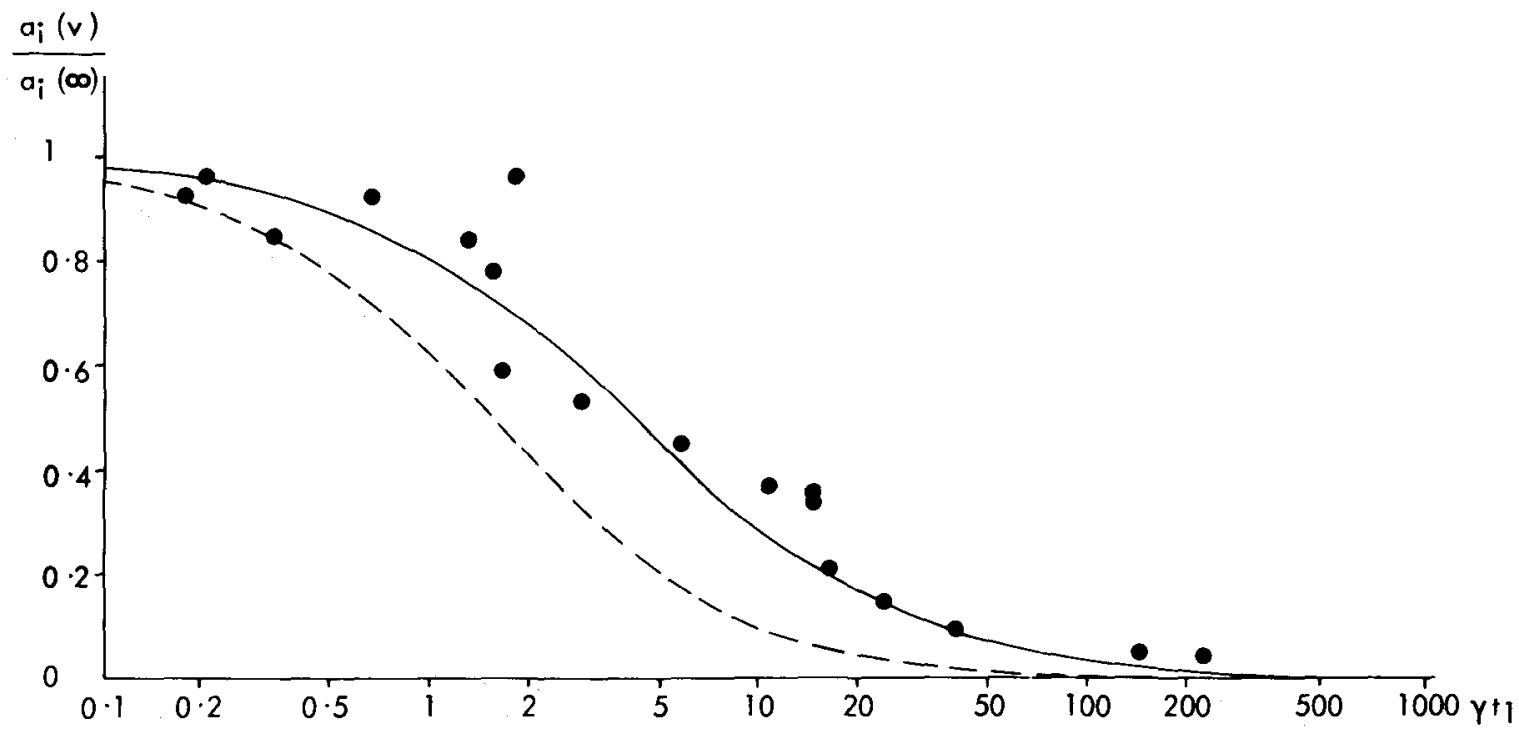

Fig. 5 The 'weakening' of the coefficients of the exponential model as a function of ${ }^{\prime} t_{1}$, the product of relaxation constant and time needed to reach the specified strain $\varepsilon_{0}$. Dots represent measured values, broken line represents the approximate expression derived previously (eqn. 15), full line represents the new expression (eqn.17)

dependence (eqn. 16), i.e. that all Maxwell elements have the same $\beta$ factor, and $\varepsilon_{0}$ was the same for all measurements, it follows from eqn. 17 that the 'factor' $\frac{\sigma\left(t_{1}\right)}{\sigma_{0}\left(\varepsilon_{0}\right)}$ is an unique function of $\gamma^{t_{1}}$. This factor can be calculated from the data of Table 2 by dividing $\frac{A_{i}}{A_{0}}$ (at a strain rate $v$ ) by $\frac{A_{i}}{A_{0}}$ (at $v=\infty$ ). In this way all results of Table 2 were represented as dots in one graph, with $\frac{A_{i}(v)}{A_{i}(\infty)}$ on the vertical axis and $\gamma t_{1}$, calculated from Table 1 and $t_{1}=\frac{\varepsilon_{0}}{v}$ on the horizontal axis (see Fig. 5). seen that this does not agree with the observed variation at all well.

The full line represents eqn. 17 , with $\beta \varepsilon_{0}=3$. This value was estimated by calculating the value of $\beta \varepsilon_{0}$ needed to give an exact fit for some points with the highest value of $\gamma t_{1}$. Since $\varepsilon_{0}=0.5$ in these experiments, this yields $\beta=6 \cdot 0$, which agrees very well with values found previously for the slowest term of the biexponentially increasing elastic modulus $\left(\beta_{2}=5 \cdot 74\right.$, vAN MASTRIGT et al., 1978). Since the full line seems to yield a reasonable fit also for experimental points at lower values of $\gamma t_{1}$, it appears that the faster exponential term of the elastic modulus is not relevant in this respect. 


\section{Discussion and conclusion}

It may be concluded from Fig. 5 that eqn. 17, with $\beta=6$, yields a good description of the non-linear dependence of the viscoelastic response of pig urinary bladder wall on strain rate. Since in the calculations no new parameters were added to the viscoelastic model described previously (VAN MASTRIGT et al., 1978), while new data could be described with parameter values found before, these findings seem to provide additional support for the model. In the abovementioned publication a discrepancy was noted between the amount of relaxation observed ("up to $50 \%$ ) and that expected on the basis of eqn. $15(10 \%)$ in measurements at very low strain rates $\left(0.0016 \mathrm{~mm} \mathrm{~s}^{-1}\right)$ and very high strains $\left(\varepsilon_{0}=4 \cdot 7\right)$. Using eqn. 17 with $\beta=6$ and the relaxation constants and coefficients of Tables 1 and 2 at $v=0.015 \mathrm{~mm} \mathrm{~s}^{-1}$, we calculate a relaxation of $42 \%$ which seems excellent agreement in view of the experimental accuracy obtained.

Eqn. 17 would thus seem to be suitable for comparison of the results of measurements of the viscoelastic response of the urinary bladder at different strain rates.

\section{References}

Coolsaet, B. L. R. A., van Duyl, W. A., van Mastrigt, R. and VAN DER ZWART, A. (1973) Stepwise cystometry of urinary bladder. Urology, II (3), 255-257.

COOlsaet, B. L. R. A., van Duyl, W. A., van Mastrigt, R. and Schouten, J. W. (1975) Viscoelastic properties of bladder wall strips. Investigative Urology, 12 (5), 351-356.

Coolsaet, B. L. R. A., van Mastrigt, R., van Duyl, W. A. and HUyGen, R. E. F. (1976) Viscoelastic properties of bladder wall strips at constant elongation. Investigative Urology, 13 (6), 435-440.

VAN MASTRIGt, R. (1977a) Constant step approximation of multi-exponential signals using a least-squares criterion. Comput. Biol. Med., 7, 231-247.

VAN MASTRIGT, R. (1977b) A systems approach to the passive properties of the urinary bladder in the collection phase. Thesis, Erasmus University Rotterdam, Rotterdam.

van Mastrigt, R., CoOlsaet, B. L. R. A. and Van DuYl, W. A. (1978) Passive properties of the urinary bladder in the collection phase. Med.\& Biol. Eng. \& Comput., 16 (5), 471482 